\title{
Effect of Glucose, Ammonium and Media Maintenance on the Time of Conidiophore Initiation by Surface Colonies of Aspergillus nidulans
}

\author{
By M. PASTUSHOK AND D. E. AXELROD \\ Department of Biological Sciences, Douglass College, Rutgers University, \\ New Brunswick, New Jersey 08903, U.S.A.
}

(Received 14 October 1975)

INTRODUCTION

Spore-derived surface colonies of Aspergillus nidulans initiate conidiophore and spore development only after a $24 \mathrm{~h}$ period of vegetative growth (Axelrod, I972). In this communication we consider why surface colonies require this $24 \mathrm{~h}$ period of vegetative growth before initiating development.

Submerged colonies of Aspergillus will initiate development only after they have depleted limiting nutrients which repress development (Galbraith \& Smith, 1969). Development of submerged colonies can follow after exhaustion of either carbon or nitrogen sources. We have therefore tested the hypothesis that surface colonies also initiate development only after depleting limiting nutrients. If the period of vegetative growth of surface colonies is due to repression by nutrients then this period would be altered by altering the medium composition; but if repression is not the cause, then the period should be unaffected by changes in medium composition.

\section{METHODS}

Organism. A prototroph of Aspergillus nidulans strain FGSC4 was used in all experiments. Media. A defined nitrate-less medium containing $1 \%(w / v)$ glucose as a carbon source, and Io $\mathrm{mM}$ L-glutamic acid (monosodium salt) as a nitrogen source was used as the basic medium. Its composition has been described previously (Gealt \& Axelrod, 1974). Where indicated, the glucose concentration was $0.03 \%(w / v)$ instead of $\mathrm{r} \%(\mathrm{w} / \mathrm{v})$, and $40 \mathrm{~mm}$ $\mathrm{NH}_{4} \mathrm{Cl}$ was substituted for glutamate as a nitrogen source. The media were solidified with I. $5 \%$ agar, or used to saturate absorbent pads supporting sterile membrane filters (Axelrod, Gealt \& Pastushok, 1973).

Kinetics of development. Diluted spore suspensions were placed on the surface of agar medium, or membrane filters, incubated at $37^{\circ} \mathrm{C}$, and the resulting number of conidiophores per colony was determined using a $\times 40$ binocular microscope (Axelrod, 1972). The average number of conidiophores per colony was determined for at least 60 colonies from three or more plates. Each experiment was repeated at least three times.

\section{RESULTS}

Glucose concentrations of $\mathrm{I} \%$, and above, and ammonium concentrations of Io $\mathrm{mM}$ and above, are known to repress several enzyme activities in this organism; whereas with glucose concentrations below $0 \cdot 1 \%$, and glutamate as nitrogen source, no such repression occurs (Arst \& Cove, 1973; Arst \& MacDonald 1975; Bartnik, Weglenski \& Protrowska, 
Table I. Effect of carbon and nitrogen sources on conidiophore development

The time of conidiophore initiation by spore-derived colonies grown on defined medium (A), is taken as the time when there is one conidiophore per colony as shown in Fig. I. The rate of conidiophore production by colonies grown on defined medium (B), is indicated by the time interval required to double the number of conidiophores per colony after initiation.

Nitrogen source

$\overbrace{0.03 \% \text { glucose }}^{\text {Carbon source }}$

A. Time of conidiophore initiation Io mM-glutamate $40 \mathrm{~mm}-\mathrm{NH}_{4} \mathrm{Cl}$

$23 \cdot 3$, S.D. $\pm 0.3 \mathrm{~h}$

$23 \cdot 2$, s.D. $\pm 0.3 \mathrm{~h}$

B. Rate of conidiophore production Io mM-glutamate $40 \mathrm{mM}^{-} \mathrm{NH}_{4} \mathrm{Cl}$
$54 \mathrm{~min}$

$54 \mathrm{~min}$
$24 \cdot 4$, S.D. $\pm \mathrm{I} \cdot \mathrm{O} \mathrm{h}$

24.4 , S.D. $\pm \mathrm{I} \cdot \mathrm{O} \mathrm{h}$

$54 \mathrm{~min}$ $30 \mathrm{~min}$

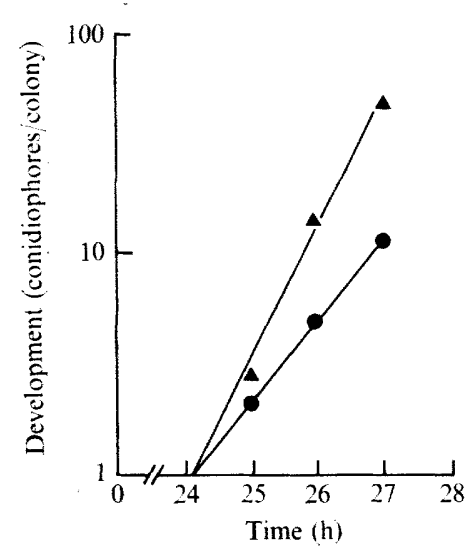

Fig. I. Kinetics of conidiophore development by spore-derived colonies grown on defined agar medium with $1 \%(\mathrm{w} / \mathrm{v})$ glucose as a carbon source and either $10 \mathrm{~mm}$-glutamate $(0)$, or $40 \mathrm{~mm}$ ammonium chloride $(\boldsymbol{\Lambda})$ as a nitrogen source.

1973; Cohen, 1973; Kinghorn \& Pateman, 1974; Roberts, I963). We have confirmed that $\mathrm{I} \%$ glucose and $40 \mathrm{~mm}-\mathrm{NH}_{4} \mathrm{Cl}$ are effective in repressing extracellular protease under the conditions in which we measured conidiophore development. However, preliminary experiments revealed that glucose concentrations between $0.0 \mathrm{I}$ and $3 \%$ did not affect the period of vegetative growth before surface colonies initiated conidiophore development.

In order to investigate this apparent lack of medium effect, the complete kinetics of conidiophore development were determined for spore-derived colonies grown on either $0.03 \%$ glucose or $1 \%$ glucose as the carbon source, and either $10 \mathrm{~mm}$-glutamate or $40 \mathrm{~mm}-\mathrm{NH}_{4} \mathrm{Cl}$ as the nitrogen source. The results in Table I A show that the time required for spore-derived surface colonies to initiate conidiophore development was not affected by any of these combinations of glucose concentration and nitrogen source. However, the rate of conidiophore production after initiation was affected by certain combinations (Table I B). The two parameters of development: (i) time required to initiate conidiophore development, and (ii) subsequent rate of conidiophore development, could be affected independently (Fig. I).

The observation that changes in medium composition can affect the two parameters of development differently may explain why some investigators have reported large effects of 
medium components on the development of certain fungi (Niederpruem \& Wessels, 1969; Turian, 1973) and others have reported little or no effects on the same fungi (Schwalb, 1971; Sargent \& Kaltenborn, 1972).

In order to determine if depletion of some nutrient other than carbon or nitrogen is necessary to initiate conidiophore development, we maintained initial concentrations of medium components by growing colonies on membrane filters and transferring them hourly to fresh medium $(3.6 \mathrm{ml})$. Colonies grown on filters without transfer initiated conidiophore development at $3 \mathrm{I} \cdot \mathrm{I}, \mathrm{S}$.D. $\pm \mathrm{I} \cdot 3 \mathrm{~h}$, and colonies transferred to fresh medium initiated conidiophore development at 27.4, S.D. $\pm 2.4 \mathrm{~h}$. Since a continuous supply of fresh medium did not prevent initiation of conidiophore development, we conclude that depletion of nutrients was not necessary for surface colonies to initiate conidiophore development.

\section{DISCUSSION}

We have shown that the period of vegetative growth of surface colonies preceding initiation of conidiophore development is not altered by either glucose concentrations or nitrogen sources that can affect enzyme activities. In addition depletion of nutrients is not necessary to initiate development of surface colonies. We now come back to the question of why surface colonies require a $24 \mathrm{~h}$ period of vegetative growth before initiating development. We have previously reported a gene-controlled activity which is necessary for the acquisition of competence to initiate development (Axelrod et al., 1973; Gealt \& Axelrod, 1974). We suggest that the long time period of vegetative growth of surface colonies before initiation of development indicates the time required to complete this endogenous genecontrolled activity, and not that required to deplete exogenous nutrients.

This work was supported by grant GMI8310 from the U.S. Public Health Service, and a grant from the Rugters Research Council.

\section{REFERENCES}

ARST, H. N. $\mathrm{J}_{\mathrm{R}}$, \& CovE, D. J. (1973). Nitrogen metabolite repression in Aspergillus nidulans. Molecular and General Genetics 126, 1 I I-14I.

ARst, H. H. \& MACDonald, D. W. (1975). A gene cluster in Aspergillus nidulans with an internally located cis-acting regulatory region. Nature, London 254, 26-3I.

AXELROD, D. E. (1972). Kinetics of differentiation of conidiophores and conidia by colonies of Aspergillus nidulans. Journal of General Microbiology 73, $18 \mathrm{I}-\mathrm{I} 84$.

Axelrod, D. E., Gealt, M. \& PastushoK, M. (1973). Gene control of developmental competence in Aspergillus nidulans. Developmental Biology 34, 9-I 5.

BartniK, E., Weglenski, P. \& ProtrowsKa, M. (1973). Ammonium and glucose repression of the arginine catabolite enzymes in Aspergillus nidulans. Molecular and General Genetics 126, 75-84.

CoHEN, B. L. (1973). Regulation of intracellular and extracellular neutral and alkaline proteases in Aspergillus nidulans. Journal of General Microbiology 79, 3I I-320.

Galbraith, J. C. \& Smith, J. E. (1969). Sporulation of Aspergillus niger in submerged culture. Journal of General Microbiology 59, 3I-45.

Gealt, M. A. \& AXelrod, D. E. (1974). Coordinate regulation of enzyme inducibility and developmental competence in Aspergillus nidulans. Developmental Biology 4I, 224-232.

Kinghorn, J. R. \& Pateman, J. A. (1974). The effect of the carbon source on ammonium regulation in Aspergillus nidulans. Molecular and General Genetics 128, 95-98.

NiederPRUEM, D. J. \& Wessels, J. G. H. (1969). Cytodifferentiation and morphogenesis in Schizophyllum commune. Bacteriological Reviews 33, 505-535.

RoBERTS, C. F. (1963). The adaptive metabolism of D-galactose in Aspergillus nidulans. Journal of General Microbiology 31, 285-295. 
Sargent, M. L. \& Kaltenborn, S. H. (1972). Effects of medium composition and carbon dioxide on circadian conidiation in Neurospora. Plant Physiology 50, 171-1 75.

Schwalb, M. N. (I97I). Commitment to fruiting in synchronously developing cultures of the basidiomycete Schizophyllum commune. Archiv für Mikrobiologie 79, 102-107.

Turian, G. (1973). Induction of conidium formation in Neurospora by lifting of catabolite repression. Journal of General Microbiology 79, 347-350. 